\title{
Real Time Sobel Square Edge Detector for Night Vision Analysis
}

\author{
Ching Wei Wang \\ Vision and Artificial Intelligence Group, \\ Department of Computing and Informatics, \\ University of Lincoln, Brayford Pool, \\ Lincoln LN6 7TS, United Kingdom \\ cweiwang@lincoln.ac.uk
}

\begin{abstract}
Vision analysis with low or no illumination is gaining more and more attention recently, especially in the fields of security surveillance and medical diagnosis. In this paper, a real time sobel square edge detector is developed as a vision enhancer in order to render clear shapes of object in targeting scenes, allowing further analysis such as object or human detection, object or human tracking, human behavior recognition, and identification on abnormal scenes or activities. The method is optimized for real time applications and compared with existing edge detectors. Program codes are illustrated in the content and the results show that the proposed algorithm is promising to generate clear vision data with low noise.
\end{abstract}

\section{Introduction}

Night vision analysis is increasingly seen as important both for academic research and industrial development. Applications involving surveillance system and medical diagnosis are obliged to perform video monitoring under circumstances of low illumination or a complete lack of visible light, in order to comply with security or medical requirements. Thermal imaging and infrared imaging techniques are generally utilized for assisting capture of video information for such applications. Due to the high price of thermal cameras and the limitation on the nature of targets, which are with or without heat, infrared imaging is more popularly adopted.

Although night vision devices improve vision capability, low illumination from limited infrared lighting is still a critical constraint for video monitoring and analysis. In addition, infrared content is constrained in grayscale format without color information provided. Thus, analytical methods, such as skin color models, are not applicable for video analysis using night vision devices. As a result, edge information that may be used to recover shapes of objects is suggested in this work for further analysis, i.e. object detection, object tracking, human activity recognition, and scene identification.

Fig. 1 presents three main functions of image processing, namely data cleaning, data transformation and data improvement, within the system flow in computer vision systems. As edge detector significantly reduces the amount of data and filters out useless information while preserving the important structural properties in an 


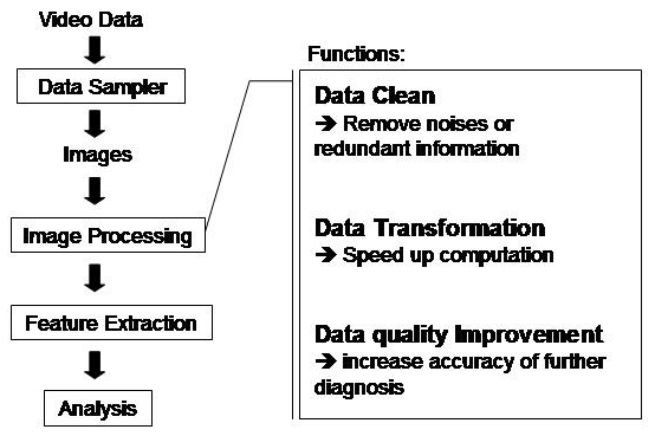

Fig. 1. Main Functions of Image Processing

image, it is thus broadly utilized for fast comparison, compression and speeding up analysis like pattern recognition. In this work, an improved edge detector is specifically developed for night vision analysis in order to extract important information in targeting scenes. Four popular adopted edge detection algorithms [1], i.e. Sobel Kernels, Prewitt Kernels, Kirsch Compass Kernels, Laplacian, and one quick edge detector, which is constructed in this work for experiments, are compared with the proposed approach, and the evaluation results show that the proposed method greatly outperforms others from processing speed, data enhancement and data cleaning viewpoints. The infrared video frames were acquired with resolution of $320 * 240$ and grayscale pixel format. The algorithm is optimized to process video in real time, i.e. 0.03 second per frame. The system is tested over a PC with CPU P4-2.4G and 256M Ram. The prototype is implemented in C\#, a high level language for fast development.

Furthermore, the improved edge detector is implemented and applied to an artificial intelligent vision analysis system [2], which monitors patients' behavior during sleep. The diagnosis is generally performed without visible light, providing a comfortable environment for patients. It shows very positive results for human activity recognition.

The paper is organized as follows. Related work and the technical challenges are presented in Section 2. After explaining the details of the existing edge detection algorithms in Section 3, the proposed edge detector algorithm, resulting images and program code are described in Section 4. Finally, future development follows in Section 5 .

\section{Related Work}

Infrared imaging and thermal imaging play important roles for night vision analysis. Under relatively low illumination levels, night vision devices provide enormous benefits for many operational environments. Security Surveillance detects intruders and 
abnormal events in a 24 hours basis; car night vision assists human driving safety; military targeting acquires specific objects' locations; medical diagnosis monitors patients' behavior without visible lights.

Plenty of research works have been devoted to human activity recognition, human detection, scene identification, object tracking and abnormal moving direction detection $[3,4,5]$. However, the performance of most methods is greatly influenced by the illumination level of the environment, contributing to unreliable and instable system performance. There is therefore a need for more robotic methods generating highquality night vision data for further analysis.

\subsection{Technical Challenges and Analysis}

The limitation of night vision using infrared cameras is that no color information is provided. Images are rendered in grayscale format. In addition, the length of the illumination of the infrared lights is also constrained, contributing to low visibility in interested regions, which diminishes the data quality for further analysis. In other words, for general grayscale images in ordinary lighting status, the range of each pixel value is within 0 to 255 . However, under low illumination, vision data is constrained into a small range, i.e. 0 to 30 . Information in the targeting scenes is reduced and shrinks into a smaller scope. As a result, a sophisticated image processing technique is necessary here as a vision enhancer and further extract useful information from the reduced-quality data. Fig.2 illustrates that each pixel value of the infrared content shrinks into a smaller range, contributing to data in lower level contrast. Thus, to overcome poor visibility of infrared content, the main idea of this work is to exaggerate differences of each pixel.

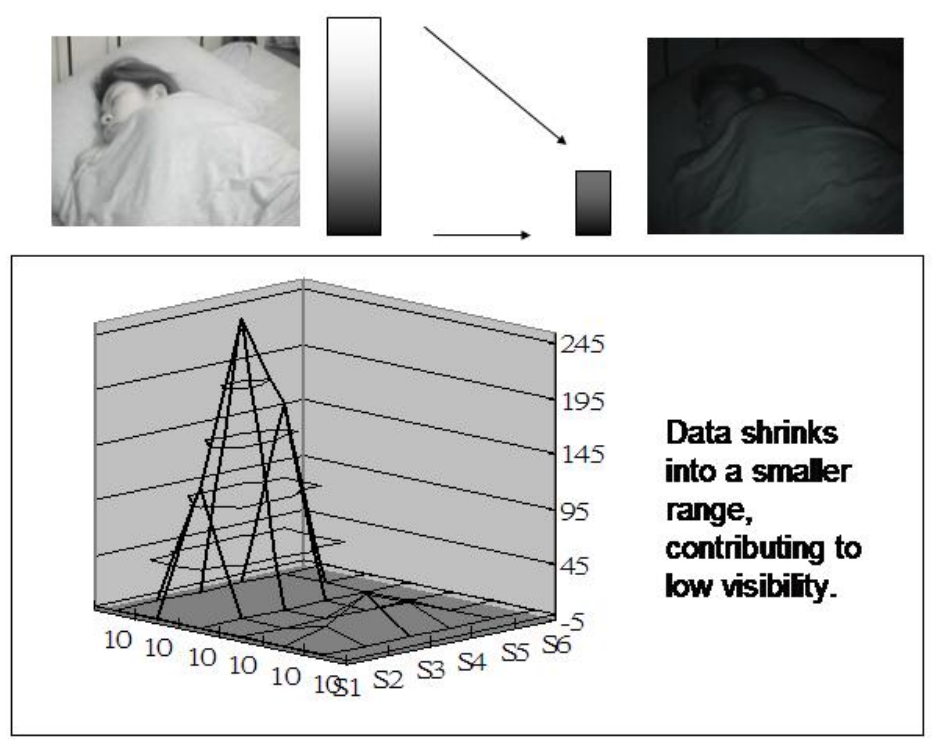

Fig. 3. General Lighting versus Low Illumination 


\section{Existing Edge Detectors}

In this work, several popular edge detectors [1] are built and compared, including first-derivative methods, such as Kirsch compass gradient kernels, Sobel kernels, Prewitt kernels, and a second derivative method, i.e. Laplacian. In this section, existing edge detectors are firstly introduced and then a short discussion is presented on the shortages of these approaches for night vision data.

Edge Detector. Let $f(x, y)$ be the image intensity function. It has derivatives in all directions. The gradient is a vector pointing in the direction in which the first derivative is highest, and whose length is the magnitude of the first derivative in that direction. If $\mathrm{f}$ is continuous and differentiable, then its gradient can be determined from the directional derivatives in any two orthogonal directions - standard to use $\mathrm{x}$ and $\mathrm{y}$.

$$
\begin{gathered}
\text { gradient }=\left[\begin{array}{l}
\frac{\partial f}{\partial x} \\
\frac{\partial f}{\partial y}
\end{array}\right] \\
\text { magnitude }=\left(\frac{\partial^{2} f}{\partial x^{2}}+\frac{\partial^{2} f}{\partial y^{2}}\right)^{\frac{1}{2}} \\
\text { direction }=\arctan \left[\begin{array}{l}
\frac{\partial f}{\partial y} \\
\frac{\partial f}{\partial x}
\end{array}\right]
\end{gathered}
$$

\subsection{Kirsch Compass Kernels}

Given k operators, $g_{k}$ is the image obtained by convolving $f(x, y)$ with the k-th operator. The $\mathrm{k}$ defines the edge direction. The gradient is defined as:

$$
\begin{aligned}
& g(x, y)=\max _{k} g_{k}(x, y) \\
& {\left[\begin{array}{rrr}
1 & 1 & 1 \\
0 & 0 & 0 \\
-1 & -1 & -1
\end{array}\right]\left[\begin{array}{rrr}
1 & 1 & 0 \\
1 & 0 & -1 \\
0 & -1 & -1
\end{array}\right]\left[\begin{array}{rrr}
1 & 0 & -1 \\
1 & 0 & -1 \\
1 & 0 & -1
\end{array}\right]\left[\begin{array}{rrr}
0 & -1 & -1 \\
1 & 0 & -1 \\
1 & 1 & 0
\end{array}\right]} \\
& \uparrow \mathrm{N} \quad \mathrm{NW} \quad \mathrm{W} \quad \measuredangle \mathrm{SW}
\end{aligned}
$$

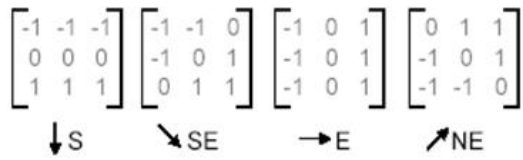

Fig. 4. Kirsch Compass Operators 


\subsection{Prewitt Kernels}

The Prewitt edge detection masks are one of the oldest and best understood methods of detecting edges in images. Basically, there are two masks, one for detecting image derivatives in $\mathrm{X}$ and one for detecting image derivatives in $\mathrm{Y}$. To find edges, a user convolves an image with both masks, producing two derivative images (dx \& dy). The strength of the edge at any given image location is then the square root of the sum of the squares of these two derivatives. The orientation of the edge is the arc tangent of $d y / d x$.

\subsection{Sobel Kernels}

Sobel detector is similar to the Prewitt detector. They both rely on central differences, but greater weights are given to the central pixels in Sobel Kernels. The Sobel kernels can also be thought of as $3 * 3$ approximations to first-derivative-of-Gaussian kernels. The equations of sobel kernels are presented below.

$$
\begin{aligned}
& G x(x, y)=[f(x+1, y-1)+2 * f(x+1, y)+f(x+1, y+1) \\
& -f(x-1, y-1)-2 * f(x-1, y)-f(x-1, y+1)] / 4 \\
& G y(x, y)=[f(x-1, y+1)+2 * f(x, y+1)+f(x+1, y+1) \\
& -f(x-1, y-1)-2 * f(x, y-1)-f(x+1, y-1)] / 4 \\
& f(x, y)=\sqrt{G x(x, y)^{2}+G y(x, y)^{2}}
\end{aligned}
$$

$$
\begin{array}{|r|r|r|}
\hline-1 & -1 & -1 \\
\hline 0 & 0 & 0 \\
\hline 1 & 1 & 1 \\
\hline
\end{array}
$$$$
\partial / \partial x
$$

Sobel Kernels:

$$
\begin{array}{|l|l|l|}
\hline-1 & 0 & 1 \\
\hline-1 & 0 & 1 \\
\hline-1 & 0 & 1 \\
\hline
\end{array}
$$$$
\partial / \partial y
$$

$$
\begin{array}{|r|r|r|}
\hline-1 & 0 & 1 \\
\hline-2 & 0 & 2 \\
\hline-1 & 0 & 1 \\
\hline 0
\end{array} \quad \begin{array}{r|r|r|}
\hline 1 & 2 & 1 \\
\hline 0 & 0 & 0 \\
\hline-1 & -2 & 1 \\
\hline
\end{array}
$$

Fig. 5. Templates to calculate the gradient value

\subsection{Laplacian}

The Laplacian $L(x, y)$ of an image with pixel intensity values $I(x, y)$ is given by:

$$
I(x, y)=\frac{\partial^{2} I}{\partial x^{2}}+\frac{\partial^{2} I}{\partial y^{2}}
$$

The equation is derived from the following equations. 


$$
\begin{aligned}
& \frac{\partial f^{2}(x, y)}{\partial^{2} x}=f^{\prime \prime}(x, y)=f^{\prime}(x+1, y)-f^{\prime}(x, y) \\
& =[f(x+1, y)-f(x, y)]-[f(x, y)-f(x-1, y)] \\
& =f(x+1, y)-2 f(x, y)+f(x-1, y) \\
& \frac{\partial f^{2}(x, y)}{\partial^{2} y}=f^{\prime \prime}(x, y)=f^{\prime}(x, y+1)-f^{\prime}(x, y) \\
& =[f(x, y+1)-f(x, y)]-[f(x, y)-f(x, y-1)] \\
& =f(x, y+1)-2 f(x, y)+f(x, y-1) \\
& I(x, y)=\frac{\partial^{2} I}{\partial x^{2}}+\frac{\partial^{2} I}{\partial y^{2}}
\end{aligned}
$$

Particularly, the higher the derivative, the more sensitivity the operator has.

\begin{tabular}{|r|r|r|}
\hline 0 & 1 & 0 \\
\hline 1 & -4 & 1 \\
\hline 0 & -1 & 0 \\
\hline
\end{tabular}$\quad$\begin{tabular}{|r|r|r|}
\hline 1 & -2 & 1 \\
\hline-2 & 4 & -2 \\
\hline 1 & -2 & 1 \\
\hline-1 & -1 & -1 \\
\hline-1 & 8 & -1 \\
\hline-1 & -1 & -1 \\
\hline
\end{tabular}

Fig. 6. Three commonly used discrete approximations in Laplacian

\subsection{Simple Edge Detect}

In order to develop a suitable edge detector, a simple edge detector is also produced for experiments. It uses a convolution filter with value below.

$$
\begin{array}{|r|r|r|}
\hline-1 & -1 & -1 \\
\hline 0 & 0 & 0 \\
\hline 1 & 1 & 1 \\
\hline
\end{array}
$$

Fig. 7. Convolution filter for simple edge detect

\subsection{Shortage on Existing Edge Detectors for Night Vision Analysis}

The main issue for night vision data is that vision information is constrained into a much smaller range as demonstrated in Fig 3. Therefore, existing approaches, which are suitable for general lighting condition, are not feasible to extract enough edge information due to low differential degree among pixel datum in each frame. Although threshold mechanism is utilized as high and low pass filters to avoid noises or redundant data, the high / low pass filters cannot exaggerate the differential level among each pixel, and thus they cannot further extract important edges of objects in the targeting scenes. 


\section{Proposed Sobel Square Edge Detectors}

As data under low illumination is constrained into a smaller range, high pass filter may remove important information. In order to defeat the low data quality issue for night vision analysis, exaggeration on differential degree among data is proposed to obtain edge information from the original scenes. A simple illustration is given in Fig 8 , which shows that low pass filter is not useful whereas high pass filter removes precious data under low illumination. The proposed approach is to increasing differential degrees among data for recovering information under general lighting condition. The proposed edge detector is adapted from Sobel kernels. The original Gx and Gy are enlarged to Ax and By. In addition, $f(x, y)$ is also magnified into $F(x, y)$. Resulting images with existing approaches and proposed method are presented in Fig. 9 whereas the original image is shown in Fig. 9. It is found that the image processed from the proposed method obtains more highlighting on important edges with lowest production on redundant information. The algorithm is equipped with equation 12, 13 and 14.
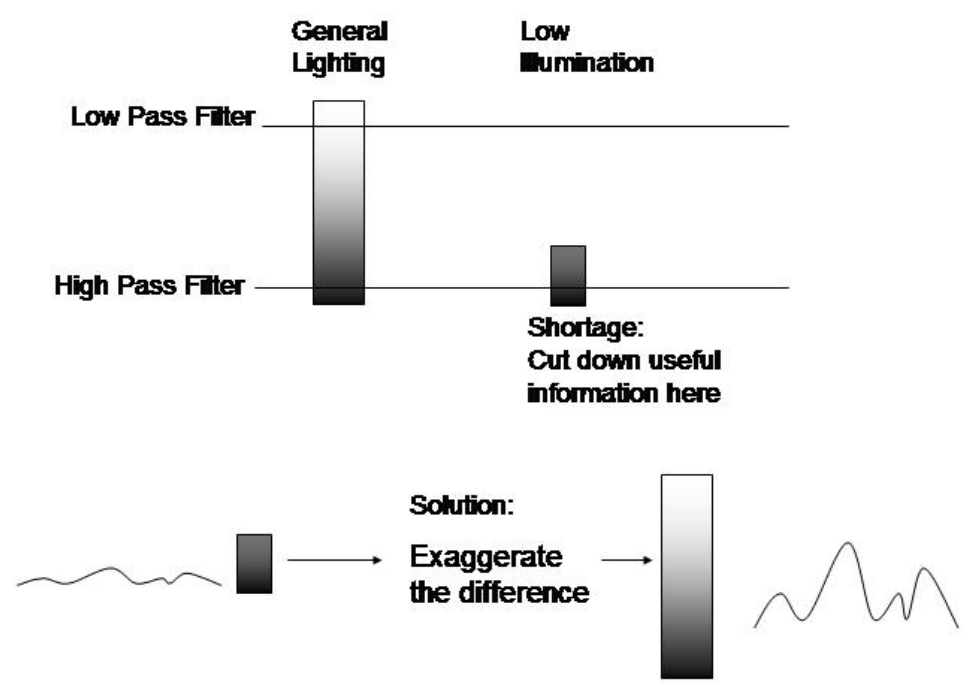

Fig. 8. Thresholds versus Exaggeration on Differences

\subsection{Proposed Method}

$$
\begin{aligned}
& A x(x, y)=f(x+1, y-1)+2 * f(x+1, y)+f(x+1, y+1) \\
& -f(x-1, y-1)-2 * f(x-1, y)-f(x-1, y+1) \\
& B y(x, y)=f(x-1, y+1)+2 * f(x, y+1)+f(x+1, y+1) \\
& -f(x-1, y-1)-2 * f(x, y-1)-f(x+1, y-1) \\
& F(x, y)=\frac{A x(x, y)^{2}+B y(x, y)^{2}}{8}
\end{aligned}
$$




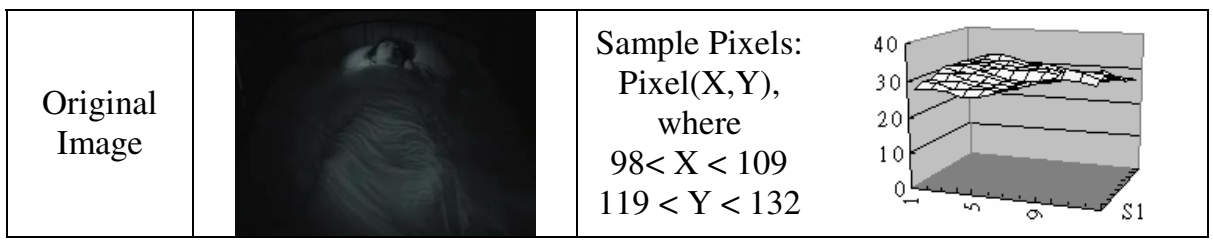

Fig. 9. Original Infrared Content

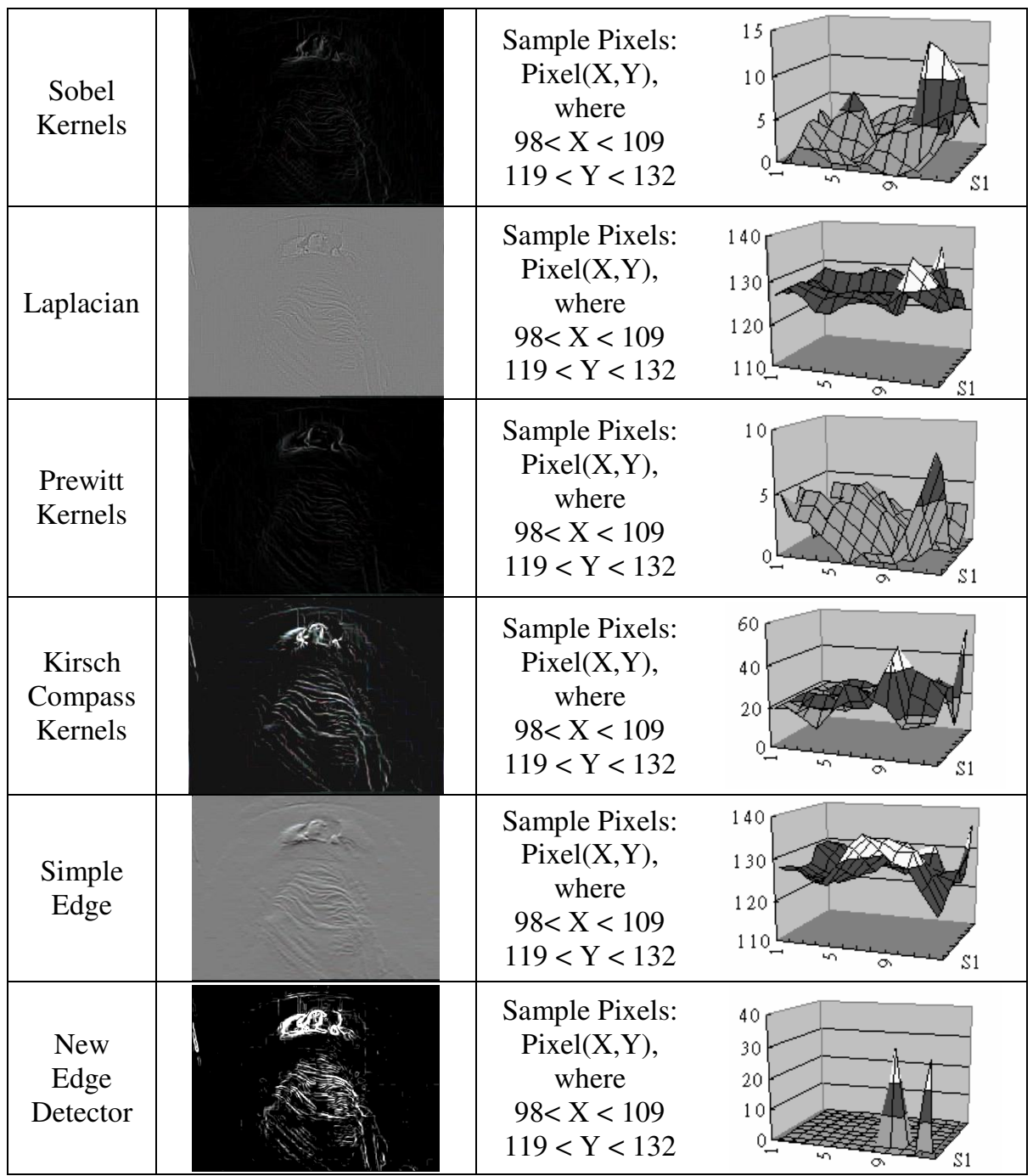

Fig. 10. Resulting Images from Different Edge Detectors 


\subsection{Resulting Images}

In this section, an original infrared image is displayed in Fig. 9 and a portion of the image is extracted and presented in a 3D chart, containing coordinates of $\mathrm{X}$ and $\mathrm{Y}$ and the pixel value of points. The image is in grayscale format, and hence the value of individual pixel is valid from 0 to 255 . Moreover, the resulting images processed by different edge detectors are shown in Fig. 10. The experimental results demonstrate that the proposed new edge detector is able to effectively extract edges and reduce redundant information.

\subsection{System Performance}

Image processing is the preliminary step for computer vision analysis, which may include more heavy computation tasks such as pattern recognition. Therefore, the preliminary process should not become the burden of overall performance both for the concerns of computation speed and CPU consumption. The system is tested over a pc with CPU P4-2.4G and 256M Ram. All algorithms are implemented in C\#, a high level language for fast development. The proposed real time sobel square is able to process 30 frames per second for real time application. For a detailed examination, it costs 0.03 second to process a $320 * 240$ frame. In comparison, existing approaches such as Sobel or Kirsch after optimization spend 0.3 second to process each frame. As a result, the proposed edge detector is suitable for applications, which take system performance as first priority. The reason why the proposed method is ten times faster than the conventional sobel detector is because the square root is removed and the number of division is reduced.

\section{Conclusion and Future Development}

The aim of this work is to provide a more robotic image processing technique in computer vision, removing constraints on night vision analysis. The proposed new edge detector appears to improve video analysis result by assisting night vision capability for diagnosis in obstructive sleep apnoea [2]. In future development, we hope that the proposed method will be applied and examined in more wide usages like surveillance system. Also, more generic algorithms are expected to be built for obtaining stable performance invariant to changes on environmental variables.

\section{Acknowledgement}

Ching Wei Wang obtains her PhD scholarship jointly funded by United Lincolnshire Hospital NHS Trust and University of Lincoln.

\section{References}

1. Lukac, R., Plataniotis, K.N., Venetsanopoulos, A.N., Bieda, R., Smolka, B.: "Color Edge Detection Techniques", Signaltheorie und Signalverarbeitung, Akustik und Sprachakustik, Informationstechnik, W.E.B. Universitat Verlag, Dresden, veol. 29, pp. 21-47, 2003 
2. Wang, C.W., Ahmed, A., Hunter, A: "Robotic Video Monitor with intelligence for diagnosis on Obstructive Sleep Apnoea". (Unpublished)

3. Lipton, A. J., Heartwell , C. H., Haering, N., and Madden, D.: "Critical Asset Protection, Perimeter Monitoring, and Threat Detection Using Automated Video Surveillance", Object Video white paper.

4. Gavrila D.M., "The visual analysis of human movement - a survey, computer vision and image understanding", vol. 73, no.1, pp.82-98, 1999

5. Zhou, J., Hoang, J.: "Real Time Robust Human Detection and Tracking System", IEEEComputer vision and pattern recognition, Volume: 3, On page(s): 149- 149, 2005

6. Desmond C. Adler, Tony H. Ko, Paul R. Herz, and James G. Fujimoto: "Optical coherence tomography contrast enhancement using spectroscopic analysis with spectral autocorrelation”, Vol. 12, No. 22 / OPTICS EXPRESS 5487, 2004 